Published in final edited form as:

Am J Cardiol. 2010 June 15; 105(12): 1661-1665. doi:10.1016/j.amjcard.2010.01.340.

\title{
Vital Exhaustion as a Risk Factor for Adverse Cardiac Events (From the Atherosclerosis Risk in Communities [ARIC] Study)
}

\author{
Janice E. Williams, PhD, MPHa , Thomas H. Mosley Jr., PhD ${ }^{b}$, Willem J. Kop, PhD ${ }^{c}$, David J. \\ Couper, PhD $^{d}$, Verna L. Welch, PhD, MPH ${ }^{e}$, and Wayne D. Rosamond, PhD $^{f}$
}

aProgram in Public Health, LaGrange College, LaGrange, Georgia bepartment of Medicine (Geriatrics), University of Mississippi Medical Center, Jackson, Mississippi 'Division of Cardiology, University of Maryland School of Medicine, Baltimore, Maryland dDepartment of Biostatistics, School of Public Health, University of North Carolina, Chapel Hill, North Carolina eDepartments of Health Policy and Management, Rollins School of Public Health, Emory University, Atlanta, Georgia fDepartment of Epidemiology, Gillings Global School of Public Health, University of North Carolina, Chapel Hill, North Carolina

\section{Abstract}

Vital exhaustion, defined as excessive fatigue, feelings of demoralization, and increased irritability, has been identified as a risk factor for incident and recurrent cardiac events, but there are no prospective studies of this association in United States samples. We examined the predictive value of vital exhaustion for incident myocardial infarction or fatal coronary heart disease (CHD) among middle-aged men and women in four US communities. Participants were 12,895 black or white men and women enrolled in the Atherosclerosis Risk In Communities (ARIC) Study cohort and followed for the occurrence of cardiac morbidity and mortality from 1990 through 2002 (maximum follow-up $=13.0$ years). Vital exhaustion was assessed using the 21-item Maastricht Questionnaire, and partitioned into approximate quartiles for statistical analyses. High vital exhaustion (the fourth quartile) predicted adverse cardiac events in age-, gender-, and race-centeradjusted analyses (1.69 [95\% C.I: 1.40 to 2.05]) and in analyses further adjusted for educational level, body mass index, plasma low density lipoprotein-and high density lipoprotein-cholesterol, systolic and diastolic blood pressure levels, diabetes mellitus, cigarette smoking status, and packyears of cigarette smoking (1.46 [95\% C.I: 1.20 to 1.79]). The risk for adverse cardiac events increased monotonically from the first through the fourth quartile of vital exhaustion. The probabilities of adverse cardiac events over time were significantly higher in people with high vital exhaustion compared to those with low exhaustion $(\mathrm{p}=0.002)$. In conclusion, vital exhaustion predicts the long-term risk for adverse cardiac events in men and women, independent of the established biomedical risk factors.

Correspondence Address: Janice E. Williams, PhD, MPH, Program in Public Health, LaGrange College, Post Office Box 3168 , LaGrange, Georgia 30241; Telephone: (706) 884-2321; jwill22@bellsouth.net.

Publisher's Disclaimer: This is a PDF file of an unedited manuscript that has been accepted for publication. As a service to our customers we are providing this early version of the manuscript. The manuscript will undergo copyediting, typesetting, and review of the resulting proof before it is published in its final citable form. Please note that during the production process errors may be discovered which could affect the content, and all legal disclaimers that apply to the journal pertain. 


\section{Keywords}

stress; risk factors; coronary disease; epidemiology

Even though support for a positive association between vital exhaustion - defined as a state of excessive fatigue, increased irritability, and demoralization - and coronary heart disease (CHD) has accumulated, most of the evidence has been derived from European populations. There are no prospective studies of vital exhaustion and acute coronary syndromes in United States samples. Using data from the Atherosclerosis Risk in Communities (ARIC) Study (1) cohort, the current analysis tested whether people with high vital exhaustion, compared to their low exhaustion counterparts, were at increased risk for incident MI and fatal CHD. The ARIC Study permitted a test of this hypothesis in a sample of middle-aged white or black men and women. We hypothesized that participants with high vital exhaustion, compared to their low exhaustion peers, would be at increased risk for adverse cardiac events and that a monotonic increase in risk from low to high exhaustion would be observed. The ARIC Study includes sufficient numbers of cardiac events to systematically examine the effects of vital exhaustion, adjusting for known predictors of cardiovascular disease progression.

\section{METHODS}

Participants in this analysis were black or white men and women, aged $48-67$, who were enrolled in the ARIC Study cohort at Visit 2 (1). ARIC is a large, population-based, prospective study of the etiology and natural history of atherosclerosis. A detailed description of study participants and research methodologies has been provided elsewhere (1). In brief, participants were selected through probability sampling from 4 U.S. communities of Washington County, Maryland; suburban Minneapolis, Minnesota; Forsyth County, North Carolina; and Jackson, Mississippi. In Jackson, Mississippi, only black participants were sampled. ARIC baseline examinations were obtained from 1987 to 1989 (Visit 1), after which participants returned at approximately three-year intervals for three follow-up clinical examinations. Hospitalizations and deaths during the follow-up period were identified through annual telephone interviews and ongoing hospital surveillance. Participants for this analysis were the 14,348 ARIC cohort members (92.2\%) who returned for the second clinic visit between 1990 and 1992 (Visit 2). Excluded from analyses were 311 participants with missing data on prevalent CHD, 761 with prevalent CHD, 38 with a racial/ethnic identity other than black or white, 46 black participants from the Washington County, Maryland and suburban Minneapolis, Minnesota field centers (hence all black participants included in these analyses were enrolled in either Jackson, Mississippi or Forsyth County, North Carolina), and 297 with incomplete responses on the vital exhaustion questionnaire, resulting in a final sample of 12,895 .

Vital exhaustion is defined as excessive fatigue, feelings of demoralization, and increased irritability and is often considered to be a form of adaptation to prolonged distress $(2,3)$. Vital exhaustion was assessed using the 21-item Maastricht Questionnaire (Appendix B) (2). Responses to the questionnaire are coded as: Yes $=2$; Don't know $=1$; No $=0$. Two items, questions 9 and 14, are reversed coded (Yes $=0$; Don't know $=1$; No $=2$ ). Responses are 
summed to obtain an overall vital exhaustion score, which ranges from $0-42$ with higher scores representing more exhaustion. Cronbach's alpha for internal consistency has been reported as 0.89 (2).

At each clinic visit, participants were evaluated on a series of physiological and behavioral parameters. Trained technicians who used standardized protocols conducted the examinations. Participants reported information regarding cigarette smoking, race/ethnicity, and level of educational attainment. Blood pressure levels were assessed using a randomzero sphygmomanometer after resting for five minutes. The average of the second and third of three consecutive measurements was used to calculate systolic and diastolic blood pressure levels. Hypertension was defined as diastolic blood pressure $\geq 90 \mathrm{mmHg}$, or systolic blood pressure $\geq 140 \mathrm{mmHg}$, or use of one or more antihypertensive medications within the past two weeks. High density lipoprotein (HDL) - cholesterol was measured enzymatically after precipitation of apolipoprotein B containing lipoproteins. Low density lipoprotein (LDL) - cholesterol was calculated using the Friedewald formula. Diabetes was defined as a fasting serum glucose level $\geq 126 \mathrm{mg} / \mathrm{dL}$, a non-fasting serum glucose level $\geq$ $200 \mathrm{mg} / \mathrm{dL}$, and/or a history of diabetes, insulin therapy, or oral hypoglycemic medication use. Body mass index (BMI) was defined as weight divided by height squared $\left(\mathrm{kg} / \mathrm{m}^{2}\right)$.

Incident cardiac events (acute myocardial infarction or fatal CHD) were ascertained in the follow-up period from the participant's clinic visit in 1990-1992 through December 31, 2002 (average $=10.9$ years; maximum $=13.0$ years). Events were identified via annual telephone interviews and hospital surveillance and validated through abstraction of the medical record and death certificates. For in-hospital events, trained abstractors obtained from the medical record presenting symptoms, ECG readings, cardiac enzyme levels, and other supportive diagnostic indicators. A maximum of three 12-lead ECG tracings were submitted to the University of Minnesota ECG Reading Center where these data were interpreted using the Minnesota code. The criteria for myocardial infarction were based on a combination of chest pain, cardiac enzymes, ECG changes, and autopsy findings. The criteria for fatal CHD were based on a combination of chest pain, underlying diagnosis from the death certificate, and other supportive diagnostic indicators from the medical record.

Additional information on out-of-hospital deaths was gathered from the decedent's next of kin, the patient's physician's and/or the coroner's or medical examiner's report. Two physicians on the ARIC Morbidity and Mortality Classification Committee reviewed the documents and assigned the diagnosis; a third panel member adjudicated any discrepancies. Detailed descriptions of the procedures used in the ascertainment of cardiac events have been reported previously (1).

Means and percentages were used to describe the population in terms of baseline CHD risk factors by approximate quartiles of vital exhaustion. One-way analyses of variance were used to examine differences in means for continuous variables across quartiles; chi-squared tests were used to examine differences in proportions for categorical variables. Cox proportional hazards regression analyses were used to assess the effects of overall vital exhaustion and component depressive affect and fatigue on risk for adverse cardiac events. The covariates in the regression analyses were: age, race-center, cigarette smoking status, pack-years of smoking, level of educational attainment, gender, BMI, systolic and diastolic 
blood pressure, plasma LDL- and HDL-cholesterol levels, and diabetes. Two models were fit, one adjusting for age, race-center, and gender and a second adjusting for all of the covariates. The Kaplan-Meier product limit method was used to estimate cumulative probabilities of adverse cardiac events over time. The log likelihood ratio test was used to assess differences in these probabilities across levels of exhaustion. All analyses were conducted using SAS version 8.2 (SAS Institute, Cary, NC).

\section{RESULTS}

In the follow-up period from 1990 to 2002 (138,435 person-years), 802 incident cardiac events occurred, equivalent to a crude rate of 5.8 per 1,000 person-years. Mean vital exhaustion scores were significantly higher in women $(12.1[\mathrm{SD}=8.9])$ than in men (7.9 $[\mathrm{SD}=7.6])(\mathrm{p}<0.0001)$, and higher in black $(12.2[\mathrm{SD}=9.1])$ than in white participants $(9.7[\mathrm{SD}=8.4])(\mathrm{p}<0.0001)$. Vital exhaustion scores were positively skewed with an overall mean of $10.30(\mathrm{SD}=8.6)$.

A baseline CHD risk factor profile indicated that participants in the highest quartile of vital exhaustion, compared to their peers in the lowest, were slightly older, heavier, and more likely to be female and black with a less than high school education. They also smoked more cigarettes, and were more likely to be diabetic and hypertensive, and to have higher systolic blood pressure and HDL-cholesterol levels. Most of these associations were markedly graded across quartiles of vital exhaustion (Table 1). Seventy-one percent of women in the cohort were postmenopausal, the majority of whom were in the lower quartiles of depressive affect. The prevalence of overall antidepressant and corticosteroid use was $4 \%$ and $1 \%$, respectively.

The highest quartile of vital exhaustion predicted adverse cardiac events in age-, gender-, and race-center-adjusted analyses (Table 2). After additional adjustment for level of educational attainment, BMI, plasma LDL-and HDL-cholesterol, systolic and diastolic blood pressure levels, cigarette smoking status, pack-years of cigarette smoking, and diabetes, the HR was attenuated, but remained statistically significant (Table 2) ( $P$ - value for linear trend $<0.0001$, using the actual vital exhaustion scores). The effect estimate was not appreciably altered when antidepressant use was added to the model [1.43 (95\% CI: 1.16 to 1.77)]. The risk for adverse cardiac events increased monotonically from the lowest to the highest quartile of vital exhaustion. Kaplan-Meier product limit estimate of the cumulative probability of adverse cardiac events is depicted in Figure 1. The multivariate-adjusted HR was 1.56 (95\% CI: 1.25 to 1.96) for the association between depressive affect and adverse cardiac events and 1.43 (95\% CI: 1.18 to 1.74 ) for fatigue.

Results of $\log$ likelihood ratio tests indicated that adverse cardiac event-free survival was significantly shorter among participants in the highest compared to the third, second and first exhaustion quartiles (Figure 1). Survival was not significantly shorter among participants in the third compared to the first $(P=0.17)$ or second $(P=0.18)$ quartiles. 


\section{DISCUSSION}

In this analysis of men and women enrolled in the ARIC cohort, vital exhaustion predicted long-term risk for incident adverse cardiac events. These findings persisted after adjustment for the traditional biomedical CHD risk factors. Sociodemographically, vitally exhausted persons were slightly older and were more likely to be female and black with a less than high school education. They also had a more adverse vascular risk factor profile. These results confirm prospective reports of a positive association between vital exhaustion and incident CHD previously observed in European samples. They indicate that excessive fatigue, feelings of demoralization, and increased irritability are important precursors to adverse cardiac events.

The biological mechanism that links vital exhaustion to adverse cardiac events is not well understood. Vital exhaustion is associated with a number of metabolic, hemodynamic, and immune responses that are known to be important for the development and progression of cardiovascular disease. Included among them are: abnormal lipid metabolism (4), decreased fibrinolysis and increased coagulation $(5,6)$, diurnal variations in fibrinolysis and coagulation (7), and low heart rate variability (8). Vital exhaustion is also associated with increased immune-mediated inflammation, which is a key factor in atherosclerosis. In a sample of healthy middle-aged men, associations between vital exhaustion, lower glucocorticoid sensitivity, and a failure of the glucocorticoids to inhibit the release of IL-6 were documented (9). In other investigations, vitally exhausted persons, compared to their nonexhausted peers, were observed to have higher levels of proinflammatory cytokines, specifically IL-6 (10), IL-1 (11), and TNF-a (12), and higher levels of C-reactive protein, a nonspecific marker of inflammation (12). The hypothalamic-pituitary adrenocortical axis (HPA), which responds to long-term stress with an attenuation of cortisol, has been implicated as an underlying mechanism (13). Support for this hypothesis has been found in studies that have documented an association of vital exhaustion with alterations in the negative feedback sensitivity of the HPA axis and suppression of HPA basal activity (14, $15)$.

Another important observation from this analysis was that vitally exhausted participants, compared to their nonexhausted peers, were more likely to be female, black and less formally educated, which collectively suggests a social disadvantage in exhaustion. Our findings are consistent with those of previous studies showing that exhaustion is commonly reported among women $(16,17)$, and with those in which women have reported greater levels of exhaustion than men $(18,19)$. It is important to note that exhaustion is a common precursor to MI among women. In a recent study, a preponderance of women (70.7\%) reported unusual fatigue as compared with a much smaller proportion (29.7\%) reporting typical angina symptoms in the prodromal period of MI (20). In the acute phase of MI, $42.9 \%$ of women reported fatigue and $54.8 \%$ reported weakness. Our findings also confirm a previously observed inverse association between exhaustion and socioeconomic status (SES) (18). However, ours is the first report of higher exhaustion in blacks compared to whites. These associations may be understood in view of the fact that people in the lower SES strata (21) and blacks (22), compared to their middle and upper SES and white counterparts, respectively, report more chronic stress. 
Vital exhaustion is closely linked to depression, as they have some common characteristics, such as fatigue and irritability. However, it is unclear whether depression and vital exhaustion are conceptually distinct or variations on a theme. Several empirical studies bear on this question, reporting differences in symptomatology (23), differences in the relationship of vital exhaustion and depression to CHD risk factors (24), yet strong positive correlations between these two constructs (25). In an investigation among patients who had undergone percutaneous coronary intervention, the effects of overall vital exhaustion and component depressive symptoms, fatigue, and hopelessness were examined as predictors of all-cause mortality and nonfatal MI (26). Depressive symptoms was the only significant predictor of adverse cardiac events in this sample of patients with established CHD, and within the depression spectrum, hopelessness was key. In the current analyses, component depressive affect and fatigue each significantly predicted adverse cardiac events and the magnitude of association was stronger for depressive affect.

Comparable relative risks have been reported for the association between clinical depression and vital exhaustion, and incident CHD. Two meta-analyses $(27,28)$ reported an overall relative risk of 1.64 for the association between depression (including clinical depression and subclinical depression) and incident CHD. When clinical depression and depressive mood were considered separately, the effect estimate was larger for clinical depression $(\mathrm{RR}=2.69)$ than depressive mood $(\mathrm{RR}=1.49)(26)$. To our knowledge, a meta-analysis of vital exhaustion has not been published. However, relative risks reported in previous studies have been comparable to those for clinical depression - being 2.07 among male college alumni (29), 2.75 among hospitalized women (30), and 2.57 and 2.51, respectively, in a community sample of women and men (18).

The strengths and weaknesses of this analysis warrant discussion. ARIC employed a wellknown, reliable instrument to assess exhaustion. However, this does not remove the potential for response biases in these self-reported data, most notably errors arising from social desirability and inaccuracies resulting from poor recall. Further, adverse cardiac events were validated using standardized criteria. In the analyses, we controlled for the effect of the established CHD risk factors on the relationship between vital exhaustion and cardiac risk, but there could have been residual confounding by unmeasured risk factors. Traditionally, black participants have been underrepresented in studies of psychosocial factors in CHD risk. The inclusion of a large bi-ethnic United States cohort of black or white men and women in the ARIC study begins to fill this gap. The prospective design of this analysis provides strong evidence of a positive association between vital exhaustion and adverse cardiac events in a population-based cohort study. Long-term follow-up permitted an examination of its effect over a 13 -year period, thus documenting long-term predictive value.

\section{ACKNOWLEDGMENTS}

The Atherosclerosis Risk in Communities (ARIC) Study is carried out as a collaborative study supported by National Heart, Lung, and Blood Institute contracts N01-HC-55015, N01-HC-55016, N01-HC-55018, N01HC-55019, N01-HC-55020, N01-HC-55021, and N01-HC-55022. The authors thank the staff and participants in the ARIC study for their important contributions. 


\section{References}

1. The ARIC Investigators. The Atherosclerosis Risk in Communities (ARIC) Study: design and objectives. Am J Epidemiol. 1989; 129:687-702. [PubMed: 2646917]

2. Appels A, Höppener P, Mulder P. A questionnaire to assess premonitory symptoms of myocardial infarction. Int J Cardiol. 1987; 17:15-24. [PubMed: 3666994]

3. Appels A, Mulder P. Excess fatigue as a precursor of myocardial infarction. Eur Heart J. 1988; 9:758-764. [PubMed: 3169045]

4. Koertge JC, Ahnve S, Schenck-Gustafsson K, Orth-Gomer K, Wamala SP. Vital exhaustion in relation to lifestyle and lipid profile in healthy women. Int J Behav Med. 2003; 10:44-55. [PubMed: 12581947]

5. Kop WJ, Hamulyak K, Pernot C, Appels A. Relationship of blood coagulation and fibrinolysis to vital exhaustion. Psychosom Med. 1998; 60:352-358. [PubMed: 9625224]

6. von Känel R, Frey K, Fischer JE. Independent relation of vital exhaustion and inflammation to fibrinolysis in apparently healthy subjects. Scand Cardiovasc J. 2004; 38:28-32. [PubMed: 15204244]

7. van Diest R, Hamulyák K, Kop WJ, van Zandvoort C, Appels A. Diurnal variations in coagulation and fibrinolysis in vital exhaustion. Psychosom Med. 2002; 64:787-792. [PubMed: 12271109]

8. Watanabe T, Sugiyama Y, Sumi Y, Watanabe M, Takeuchi K, Kobayashi F, Kono K. Effects of vital exhaustion on cardiac autonomic nervous functions assessed by heart rate variability at rest in middle-aged male workers. Int J Behav Med. 2002; 9:68-75. [PubMed: 12112997]

9. Wirtz PH, von Känel R, Schnorpfeil P, Ehlert U, Frey K, Fischer JE. Reduced glucocorticoid sensitivity of monocyte interleukin- 6 production in male industrial employees who are vitally exhausted. Psychosom Med. 2003; 65:672-678. [PubMed: 12883121]

10. Sjögren E, Leanderson P, Kristenson M, Ernerudh J. Interleukin-6 levels in relation to psychosocial factors: studies on serum, saliva, and in vitro production by blood mononuclear cells. Brain Behav Immun. 2005; 20:270-278. [PubMed: 16183246]

11. Appels A, Bär FW, Bär J, Bruggeman C, de Baets M. Inflammation, depressive symptomatology, and coronary disease. Psychosom Med. 2000; 62:601-605. [PubMed: 11020087]

12. Kop WJ, Gottdiener JS, Tangen CM, Fried LP, McBurnie MA, Walston J, Newman A, Hirsch C, Tracy RP. Inflammation and coagulation factors in persons $>65$ years of age with symptoms of depression but without evidence of myocardial ischemia. Am J Cardiol. 2002; 89:419-424. [PubMed: 11835923]

13. Melamed S, Sirom A, Toker S, Berliner S, Shapira I. Burnout and risk of cardiovascular disease: evidence, possible causal paths, and promising research directions. Psych Bull. 2006; 132:327353.

14. Bellingrath S, Weigl T, Kudielka BM. Cortisol dysregulation in school teachers in relation to burnout, vital exhaustion, and effort-reward-imbalance. Biol Psychol. 2008; 78:104-113. [PubMed: 18325655]

15. Nicolson NA, van Diest R. Salivary cortisol patterns in vital exhaustion. J Psychosom Res. 2000; 49:335-342. [PubMed: 11164057]

16. Uuskula M. Psychological differences between young male and female survivors of myocardial infarction. Psychother Psychosom. 1996; 65:327-330. [PubMed: 8946531]

17. Brezinka V, Dusseldorp E, Maes S. Gender differences in psychosocial profile at entry into cardiac rehabilitation. J Cardiopulm Rehabil. 1998; 18:445-449. [PubMed: 9857277]

18. Prescott E, Holst C, Grönbaek M, Schnohr P, Jensen G, Barefoot J. Vital exhaustion as a risk factor for ischaemic heart disease and all-cause mortality in a community sample: a prospective study of 4084 men and 5479 women in the Copenhagen City Heart Study. Int J Epidemiol. 2003; 32:990997. [PubMed: 14681262]

19. Schuitemaker GE, Dinant GJ, van der Pol GA, Appels A. Assessment of vital exhaustion and identification of subjects at increased risk of myocardial infarction in general practice. Psychosomatics. 2004; 45:414-418. [PubMed: 15345786] 
20. McSweeney JC, Cody M, O’Sullivan P, Elberson K, Moser DK, Garvin BJ. Women's early warning symptoms of acute myocardial infarction. Circulation. 2003; 108:2619-2623. [PubMed: 14597589]

21. Gallo LC, Matthews KA. Understanding the association between socioeconomic status and physical health: do negative emotions play a role? Psychological Bull. 2003; 129:10-51.

22. Troxel WM, Matthews KA, Bromberger JT, Sutton-Tyrrell K. Chronic stress burden, discrimination, and subclinical carotid artery disease in African American and Caucasian women. Health Psych. 2003; 22:300-309.

23. van Diest R, Appels A. Vital exhaustion and depression: A conceptual study. J Psychosom Res. 1991; 35:535-544. [PubMed: 1681098]

24. Kopp MS, Falger PRJ, Appels A, Sxedmak S. Depressive symptomatology and vital exhaustion are differentially related to behavioral risk factors for coronary artery disease. Psychosom Med. 1998; 60:752-758. [PubMed: 9847036]

25. Wojciechowski FL, Strik JJMH, Falger P, Lousberg R, Honig A. The Relationship between depressive and vital exhaustion symptomatology post-myocardial infarction. Acta Psychiatr Scand. 2000; 102:359-365. [PubMed: 11098808]

26. Pedersen SS, Denollet J, Daemen J, van de Sande M, de Jaegere PT, Serruys PW, Erdman RAM, van Domburg RT. Fatigue, depressive symptoms, and hopelessness as predictors of adverse clinical events following percutaneous coronary intervention with paclitaxel-eluting stents. J Psychosom Res. 2007; 62:455-461. [PubMed: 17383497]

27. Rugulies R. Depression as a predictor for coronary heart disease. Am J Prev Med. 2002; 23:51-61. [PubMed: 12093424]

28. Wulsin LR, Singal BM. Do depressive symptoms increase the risk for the onset of coronary disease? A systematic quantitative review. Psychosomatic Med. 2003; 65:201-210.

29. Cole SR, Kawachi I, Sesso HD, Paffenbarger RS, Lee I-M. Sense of exhaustion and coronary heart disease among college alumni. Am J Cardiol. 1999; 84:1401-1405. [PubMed: 10606112]

30. Appels A, Falger PRJ, Schouten EGW. Vital exhaustion as a risk indicator for myocardial infarction in women. J Psychosom Res. 1993; 37:881-890. [PubMed: 7905533]

\section{Appendix A. The Maastricht Questionnaire}

1. Do you often feel tired? (F)

2. Do you often have trouble falling asleep? (D)

3. Do you wake up repeatedly during the night? (D)

4. Do you feel weak all over? (F)

5. Do you have the feeling that you haven't been accomplishing much lately? (F)

6. Do you have the feeling that you can't cope with everyday problems as well as you used to? (F)

7. Do you believe that you have come to a "dead end"? (D)

8. Do you lately feel more listless than before? (F)

9. I enjoy sex as much as ever. (F)

10. Have you experienced a feeling of hopelessness recently? (D)

11. Does it take more time to grasp a difficult problem than it did a year ago? (F)

12. Do little things irritate you more lately than they used to?

13. Do you feel you want to give up trying? 
14. I feel fine. $(\mathrm{F})$

15. Do you sometimes feel that your body is like a battery that is losing its power? (F)

16. Would you want to be dead at times? (D)

17. Do you have the feeling these days that you just don't have what it takes any more? (F)

18. Do you feel dejected? (D)

19. Do you feel like crying sometimes? (D)

20. Do you ever wake up with a feeling of exhaustion and fatigue? (F)

21. Do you have increasing difficulty in concentrating on a single subject for long? (F)

F - Fatigue D - Depressive affect 


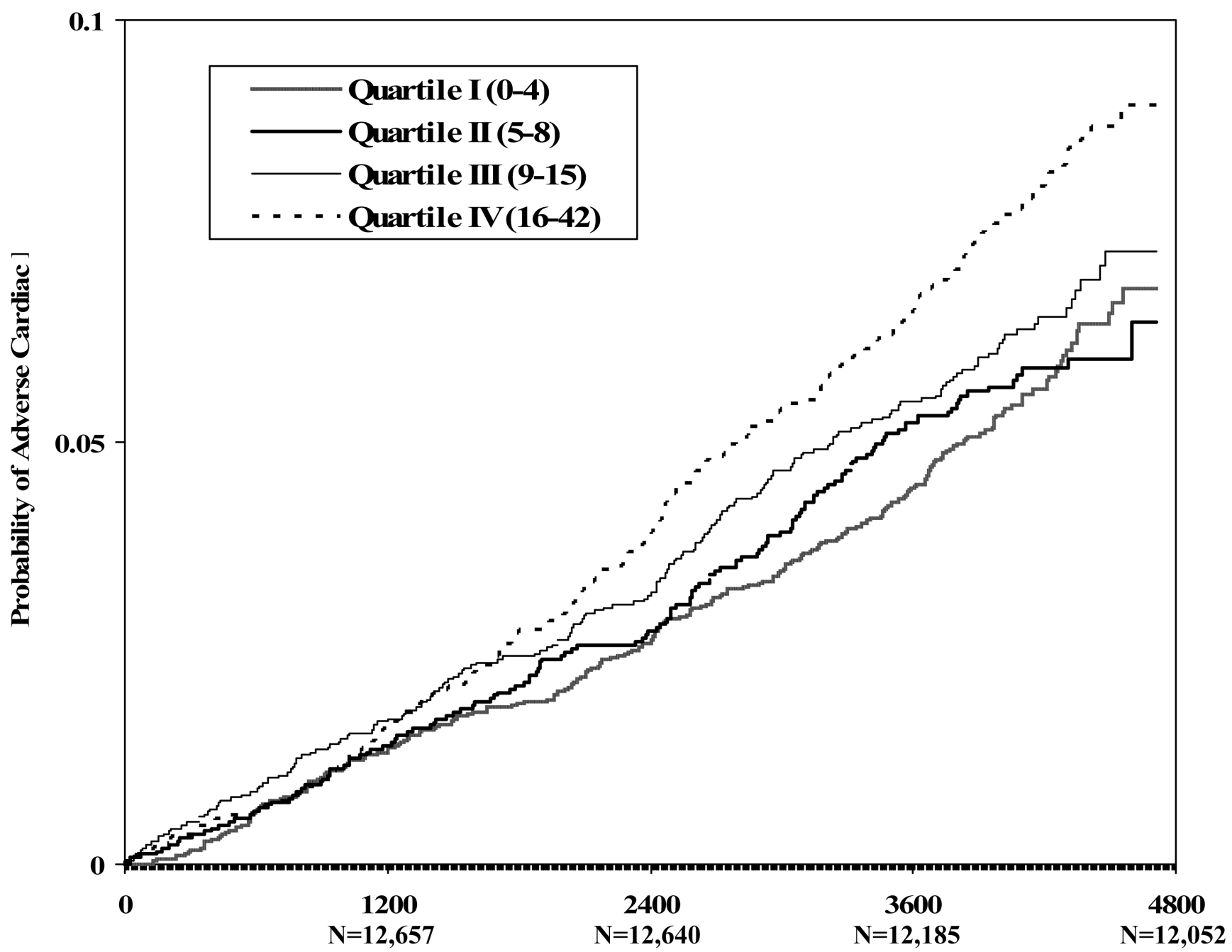

Days of follow-up

Figure 1.

The Kaplan-Meier product limit estimate of the cumulative probability of adverse cardiac events by level of vital exhaustion $(P=0.01,0.0005,0.002$ for differences between $\mathrm{Q} 4$ and Q3, Q2, and Q1, respectively). 


\section{을 \\ 졸}

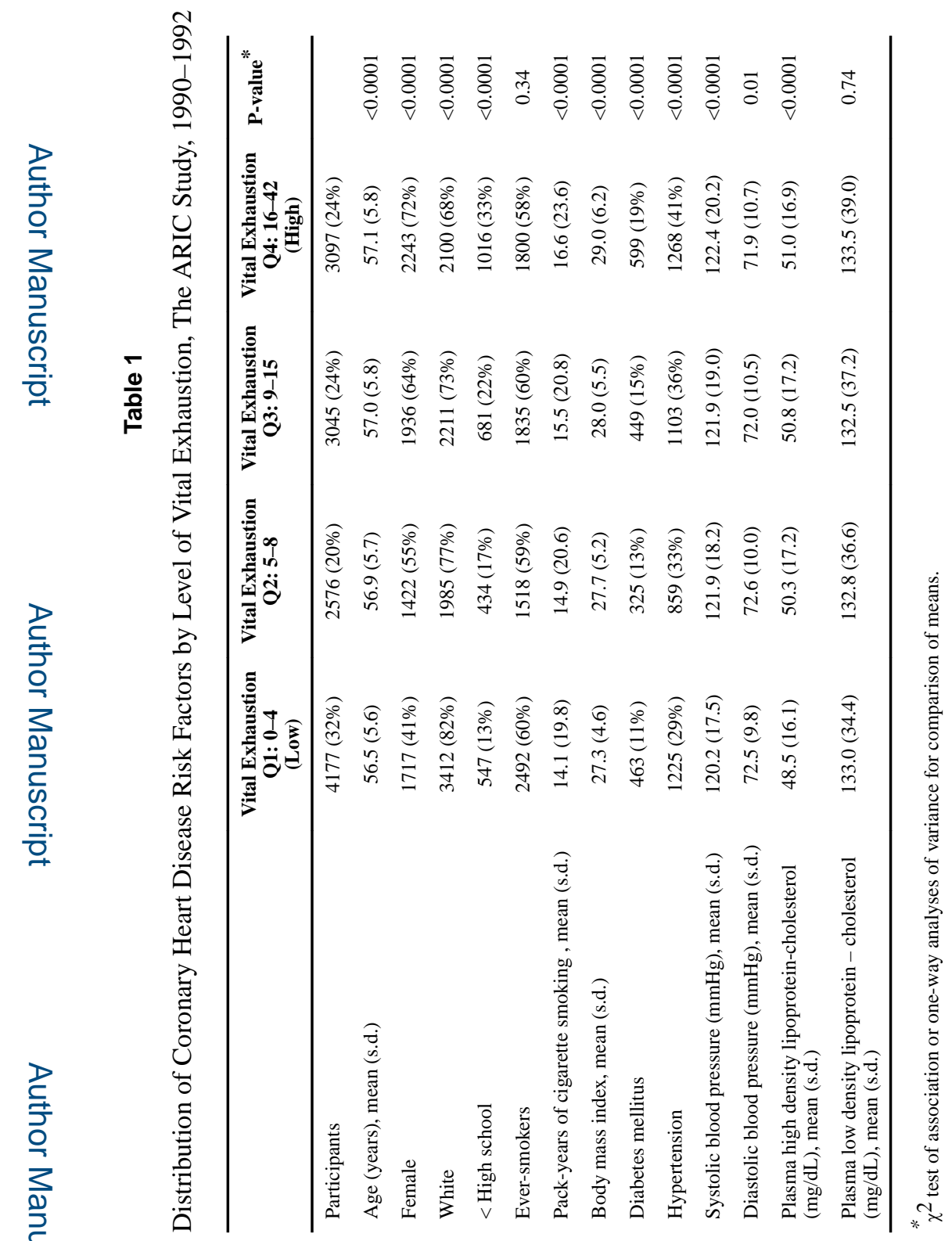

Am J Cardiol. Author manuscript; available in PMC 2016 June 30. 


\section{Table 2}

Hazard Ratios (95\% Confidence Interval) for the Association Between Vital Exhaustion and Adverse Cardiac Events, The ARIC Study, 2002

\begin{tabular}{lcc}
\hline Regression Models & & \\
\hline & Number of incident events = 800 \\
\hline Model 1 $^{a}$ & Hazard Ratios & P-value \\
Exhaustion Q1 (Reference) & 1.00 & \\
Exhaustion Q2 & $1.08(0.88-1.33)$ & 0.45 \\
Exhaustion Q3 & $1.28(1.06-1.56)$ & 0.01 \\
Exhaustion Q4 & $1.69(1.40-2.05)$ & $<0.0001$ \\
Model 2 & & \\
Exhaustion Q1 (Reference) & 1.00 & \\
Exhaustion Q2 & $1.02(0.82-1.27)$ & 0.84 \\
Exhaustion Q3 & $1.18(0.97-1.45)$ & 0.10 \\
Exhaustion Q4 & $1.46(1.20-1.79)$ & 0.0002 \\
\hline
\end{tabular}

${ }^{a}$ Adjusted for age, gender, race-center

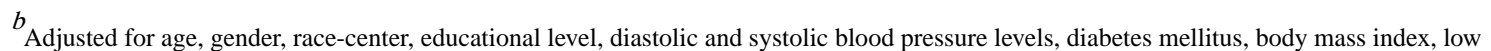
density lipoprotein- and high density lipoprotein cholesterol levels, cigarette smoking status, pack-years of cigarette smoking 